\title{
Apoptosis selectively induced in BEL-7402 cells by folic acid-modified magnetic nanoparticles combined with $100 \mathrm{~Hz}$ magnetic field
}

This article was published in the following Dove Press journal:

International Journal of Nanomedicine

23 April 2014

Number of times this article has been viewed

\author{
Jian Wen' \\ Shulian Jiang' \\ Zhiqiang Chen' \\ Wei Zhao' \\ Yongxiang $\mathrm{Yi}^{\prime}$ \\ Ruili Yang' \\ Baoan Chen ${ }^{2}$
}

'Second Affiliated Hospital of Southeast University, ${ }^{2}$ Department of Hematology, Zhongda Hospital, Medical School, Southeast University, Nanjing, People's Republic of China

Correspondence: Baoan Chen Department of Hematology, Zhongda Hospital, Medical School, Southeast University, 87 Dingjia Qiao, Nanjing Hunan Road, Nanjing 210029,

People's Republic of China

Tel +862583272006

$\mathrm{Fax}+862583272011$

Email cba8888@hotmail.com
Objective: To explore the effect of folic acid-modified magnetic nanoparticles (FA-MNPs) combined with a $100 \mathrm{~Hz}$ extremely low-frequency electromagnetic field (ELF-EMF) on the apoptosis of liver cancer BEL-7402 cells.

Materials and methods: MNPs (20 nm) were prepared by coprecipitation, and then folic acid was coated onto MNPs to prepare FA-MNPs. BEL-7402 cells and HL7702 cells were selected as liver cancer cells and normal liver cells, respectively. The ELF-EMF was generated from a solenoid coil. Cellular uptake of NPs was determined by inductively coupled plasma atomic emission spectroscopy. A 3-(4,5-dimethylthiazol-2-yl)-2,5-diphenyltetrazolium bromide assay was used to evaluate cell inhibition. Apoptosis was analyzed by flow cytometry. Statistical analyses were performed using two-way analysis of variance.

Results: FA-MNPs combined with a $100 \mathrm{~Hz}$ magnetic field significantly inhibited cell proliferation and induced higher apoptosis compared to either the ELF-EMF alone or FA-MNPs alone. FA-MNPs showed a better apoptosis effect and higher iron uptake in BEL-7402 cells compared to in HL7702 cells. On the basis of the ELF-EMF, higher doses of FA-MNPs brought higher apoptosis and higher iron uptake in either BEL-7402 cells or HL7702 cells.

Conclusion: These results suggest that FA-MNPs may induce apoptosis in a cellular iron uptake-dependent manner when combined with an ELF-EMF in BEL-7402 cells.

Keywords: extremely low-frequency magnetic field, magnetic nanoparticle, apoptosis, liver cancer, folic acid

\section{Introduction}

The most common primary malignancy of the liver in adults is hepatocellular carcinoma (HCC; or hepatoma). It is currently the fifth-most common solid tumor worldwide and the third-leading cause of cancer-related death. ${ }^{1,2}$ The prognosis of HCC is poor for most patients, because $\mathrm{HCC}$ is often diagnosed at a late stage and current treatment options are rather limited. The inherent difficulty of treating this malignancy has prompted many to consider a new therapeutic approach. ${ }^{3}$

Recently, nanoparticles (NPs) have begun to offer new opportunities in many fields. ${ }^{4}$ Magnetic NPs have emerged as a potential treatment modality for cancer therapy. $\mathrm{Fe}_{3} \mathrm{O}_{4}$ NPs are the only magnetic nanomaterials approved for clinical use by the US Food and Drug Administration, and the preparation method is also relatively simple. ${ }^{5}$ Magnetic NPs have the prospect of being used in diagnostic research for magnetic resonance, eg, $\mathrm{Fe}_{3} \mathrm{O}_{4}$ imaging. ${ }^{6}$ Additionally, magnetic NPs could enhance therapeutic effects and reduce side effects of drugs when used in combination with conventional cancer treatment. ${ }^{7}$ However, these conventional cancer treatments have cytotoxicity, and their use is limited in liver cancer treatment. 
As a potential noninvasive combination method for magnetic NPs (MNPs), electromagnetic fields (EMFs) have been employed as useful tools in medicine. Frequencies below $300 \mathrm{~Hz}$ are known as extremely low-frequency (ELF) EMFs, and they do not cause direct damage to deoxyribonucleic acid. ${ }^{8}$ These properties have led to the expansion of ELF-EMF to noncytotoxic therapeutic purposes in various diseases, including cancer. Moreover, previous experiments demonstrated that the combination of MNPs with ELF-EMF significantly induced early apoptosis, compared to MNPs or ELF-EMF alone. ${ }^{9}$ MNPs coculturing with cells had no influence on cell physiology, while ELF-EMF exposure inhibited cell proliferation, arrested cells at the $\mathrm{G}_{0} / \mathrm{G}_{1}$ period, and induced cells into early apoptosis. MNPs strengthened the effects (ie, higher cell-proliferation inhibitive ratio and higher apoptosis ratio) through influencing cell ion metabolism by strengthening the reduction of cation-exchange current on cellular membrane. ${ }^{9}$ However, unmodified MNPs could only be used in vitro, due to their inability to reach the tumor site in effective concentrations in vivo. ${ }^{10}$ Folic acid (FA)-modified NPs may be a potential alternative solution for in vivo use. FA is a water-soluble vitamin, and has been used for targeting drugs to cancer cells. Folate receptors exhibit limited expression on healthy cells, but are often present in large numbers on cancer cells. ${ }^{11}$ Folate receptor-mediated drug delivery is based on conjugation with FA, which is internalized by folate receptor-mediated endocytosis. There are also reports that FA has been immobilized on superparamagnetic particles ${ }^{12}$ and polymer NPs, ${ }^{13}$ and used for selective targeting of tumor cells and specific cellular uptake of NPs. ${ }^{14}$

This experiment was designed to explore the effect of FA-MNPs combined with ELF-EMF on the apoptosis of liver cancer BEL-7402 cells and normal liver HL7702 cells, and to further test whether its effect on these cells was related to the cellular iron uptake of FA-MNPs.

\section{Materials and methods Chemicals and reagents}

Chemicals of analytical grade and deionized water were used throughout the preparation procedure. Ferrous sulfate heptahydrate $\left(\mathrm{FeSO}_{4} \cdot 7 \mathrm{H}_{2} \mathrm{O}\right)$, ferric chloride hexahydrate $\left(\mathrm{FeCl}_{3} \cdot 6 \mathrm{H}_{2} \mathrm{O}\right)$, concentrated ammonia, and ethylenediaminetetraacetic acid (EDTA) were bought from Huifengda Chemical (Jinan, People's Republic of China). Lecithin and folic acid were bought from Dingguo Biotechnology (Nanjing, People's Republic of China). BEL-7402 cells (a human hepatoma cell line) and HL7702 cells (a normal liver cell line) were bought from the Shanghai Cell Research Center of the Chinese Academy of Sciences
(Shanghai, People's Republic of China). Roswell Park Memorial Institute (RPMI) 1640 medium, trypsin, L-glutamine, and fetal calf serum were bought from Life Technologies (Carlsbad, CA, USA). An Annexin V-FITC apoptosis-detection kit was purchased from BD Biosciences (San Jose, CA, USA).

\section{Preparation and characterization of FA-MNPs}

The superparamagnetic NPs with average size of $20 \mathrm{~nm}$ were synthesized by the following procedure. We dissolved a stoichiometric ratio 1:2 ferrous sulfate heptahydrate and ferric chloride hexahydrate deionized water under vigorous stirring to prepare a total concentration of $0.20 \mathrm{M}$ ferrite solution as an iron source. Concentrated ammonia was then dissolved in an aqueous solution to form 3.5 $\mathrm{M}$ ammonium hydroxide $\left(\mathrm{NH}_{4} \mathrm{OH}\right)$ as a base source. After that, during rigorous stirring, the mixture was titrated to have a $\mathrm{pH}$ of around $10-11$ by adding drops of $3.5 \mathrm{M}$ ammonium hydroxide at room temperature $\left(18^{\circ} \mathrm{C}-22^{\circ} \mathrm{C}\right)$. The solution became black, due to the formation of $\mathrm{Fe}_{3} \mathrm{O}_{4}$ particles. Aggregates were then removed by centrifugation at a low speed of 4,000 rpm for 5 minutes. Lecithin $(20 \mathrm{mg})$ was mixed with $50 \mathrm{~mL}$ deionized water, and the mixture was sonicated in a bath sonicator at 1,500 $\mathrm{Hz}$ for 10 minutes. It was further mixed with $5 \mathrm{mg}$ MNPs and $50 \mathrm{~mL}$ glycerol, poured into agate mortar, and ground at 20 rpm for 2 hours. The final mixture was sonicated for 3 hours and stood still, the upper-layer supernatant was decanted, and the lower-layer particles were collected and washed using deionized water three times. The lecithin-modified MNPs were then mixed with $2.0 \mathrm{mg} / \mathrm{mL}$ FA for 24 hours, sonicated in a bath sonicator at $1,500 \mathrm{~Hz}$ for 30 minutes every 2 hours, and stood still for 48 hours. A 1 T magnetostatic field was used to collect the FA-MNPs. Moreover, NP size and morphology were determined by scanning electron microscopy (SEM) and $\mathrm{X}$-ray diffraction (XRD). NP diameter was calculated by the Scheller equation:

$$
\mathrm{D}=\frac{\mathrm{K} \lambda}{\beta \cos \theta}
$$

where $\mathrm{D}$ is powder diameter; $\mathrm{K}=0.94$, shape factor; $\lambda=0.1540$, wavelength of diffraction ray; $\beta$ is peak half width; and $\theta$ is the diffraction angle.

\section{Cell culture}

BEL-7402 and HL7702 cells were cultured in RPMI 1640 medium with L-glutamine supplemented with $10 \%$ fetal calf serum and 1\% antibiotic-antimycotic solution at 
$37^{\circ} \mathrm{C}$ in a humidified atmosphere of $5 \% \mathrm{CO}_{2}$ in air. These cells grew in a monolayer and were subcultured with $0.05 \%$ trypsin and $0.02 \%$ EDTA in Dulbecco's phosphate-buffered saline (PBS). Thereafter, the cells were washed twice by centrifugation and resuspended in the complete media for reseeding and growing in new culture flasks. Cell viability was determined by staining with trypan blue, and cells were counted using a hemocytometer.

\section{Nanoparticles inside cells evaluated using flow-cytometric light-scatter analysis}

BEL-7402 cells treated with $0.25 \mathrm{mg} / \mathrm{mL}$ FA-MNPs for 24 hours were trypsinized and suspended in medium, while those BEL-7402 cells treated without FA-MNPs for 24 hours were selected as the control. The amount of particles taken up by the cells was analyzed by flow cytometry (FCM) (BD Biosciences). In FCM, the laser beam (488 nm) illuminates cells in the sample stream, which goes through the sensing area. The laser light scattered at narrow angles to the axis of the laser beam is called forward-scattered light. The laser light scattered at about a $90^{\circ}$ angle to the axis of the laser beam is called side-scattered (SS) light. The intensities of SS light are proportional to the intracellular density and its related cellular particle uptake. By comparing the SS light intensities of control cells without FA-MNP treatment, we were able to test whether FA-MNPs were inside cells by checking SS light intensities. ${ }^{15}$

\section{ELF-EMF treatment}

An ELF-EMF was generated from a solenoid coil with an interior diameter of $20 \mathrm{~cm}$, and regulated through a frequency transducer and transformer. In this study, a $100 \mathrm{~Hz}$ EMF sine wave with a mean intensity of $0.7 \mathrm{mT}$ was chosen for its easy access. The 24-well culture plates cultured with either BEL-7402 cells or HL7702 cells were placed inside the solenoid coils during ELF-EMF exposure, and the EMF ran perpendicular to the cell-culture surface. Each exposure was isolated to avoid interference from electronic devices, and lasted for 30 minutes while air flush continuously flowed through the exposure system to prevent a thermal effect from EMF exposure. The interval between each exposure was 1 hour. Sham EMF exposure was performed by turning off the power supply to the solenoid coil. A total of 150 minutes' EMF exposure was used in this study, for our previous study had shown that 150 minutes' exposure was easy to perform and could obtain a significant apoptosis effect when combined with MNPs. ${ }^{9}$ The temperatures of the EMF-exposure groups and the sham-exposure groups were measured.

\section{Cell proliferation-inhibition analysis}

A cell-counting kit (CCK-8; Dojindo, Kumamoto, Japan) was used to measure cell proliferation. The experiments were carried according to the kit guideline. Briefly, BEL7402 cells and HL 7702 cells under the same conditions were treated initially with $0.25 \mathrm{mg} / \mathrm{mL}$ FA-MNPs for 24 hours following ELF-EMF exposure for 150 minutes, and then cells were washed and collected for proliferation-inhibition assays using the CCK-8, following the manufacturer protocol. Optical densities (ODs) were measured at $450 \mathrm{~nm}$ using a microplate reader, and the cell-inhibition ratio was calculated as $\left(\left[1-\mathrm{OD}_{\text {treated group }}\right] / \mathrm{OD}_{\text {control group }}\right) \times 100 \%$.

\section{Apoptosis analysis by flow cytometry}

Cell-apoptosis rates were measured using the Annexin V-FITC apoptosis-detection kit to identify early apoptotic cells (Annexin V-FITC-positive, PI-negative) by FCM. At least $0.4 \times 10^{4}$ cells from each sample were analyzed. Cell Quest software (BD Biosciences) was used to assess apoptosis rates. Events falling into the fluorescein isothiocyanate (FITC) ${ }^{+} \mathrm{PI}^{-}$region of the lower-right quadrant were counted as apoptotic cells. The apoptosis rates of sample cells were calculated directly from the gated histograms. Four replicates were measured, and the results were averaged with standard deviation.

To study the apoptosis effected by cell-line types when treated by FA-MNPs combined with ELF-EMF, two groups were compared: in group 1, BEL-7402 cells were cultured in media containing FA-MNPs at 0.0, 0.125, 0.25, 0.5, 1.0, and $2.0 \mathrm{mg} / \mathrm{mL}$, respectively, for 24 hours, and then treated by ELF-EMF for 150 minutes; in group 2, HL7702 cells were cultured and treated with the same conditions. Cells were then immediately collected for apoptosis analysis.

To further study the apoptosis effected by the MNPs with or without FA, two groups were compared: in group 1, BEL-7402 cells were cultured in media containing FA-MNPs at $0.125,0.25,0.5,1.0$, and $2.0 \mathrm{mg} / \mathrm{mL}$, respectively, for 24 hours, and then treated by ELF-EMF for 150 minutes; in group 2, BEL-7402 cells were cultured in media containing MNPs and treated under the same conditions. Cells were then immediately collected for apoptosis analysis.

\section{Cellular uptake of nanoparticles by inductively coupled plasma atomic emission spectroscopy}

Cells were grown in 24-well culture plates, with approximately $10^{5}$ cells $/ \mathrm{mL}$ of media to quantify intracellular uptake of NPs. After incubation at $37^{\circ} \mathrm{C}$ for 20 hours, 
the BEL-7402 cells were reseeded with the culture media containing NPs at concentrations of $0.0,0.125,0.25,0.5$, 1.0 , and $2.0 \mathrm{mg} / \mathrm{mL}$ for 24 hours, and then treated by ELFEMF for 150 minutes. Meanwhile, HL7702 cells were reseeded under the same conditions. Cell samples were collected before ELF-EMF treatment, and the intracellular iron uptake was quantified using inductively coupled plasma atomic emission spectroscopy (ICP-AES). Cells were washed with PBS, detached, resuspended, counted, and centrifuged down, and the cell pellet was dissolved in $37 \% \mathrm{HCl}$ solution at $70^{\circ} \mathrm{C}-80^{\circ} \mathrm{C}$ for 30 minutes. Four replicates were measured, and the results were averaged with standard deviation.

\section{Statistical analysis}

SPSS 13.0 (SPSS Inc., Chicago, IL, USA) was used as the statistical analysis software. Statistical analyses were performed using two-way analysis of variance, and statistical values are presented as means \pm standard error of mean. Regression analysis of curve estimation was performed to explore the relationship between apoptosis and iron uptake. Throughout, $P<0.05$ was considered significant.

\section{Results}

\section{Preparation of FA-MNPs}

SEM photos showed that the MNPs were shaped as irregular balls around $20 \mathrm{~nm}$ in diameter (Figure 1A). The XRD pattern showed that the mean diameters of the unmodified MNPs and lecithin-modified MNPs were calculated as about 20 (Figure 1D), and 26 nm (Figure 1E), respectively, similar to SEM photos. SEM images also showed that the FA coatings were on individual lecithin-modified MNPs (corresponding to the tadpole-like protuberance from the MNP cores) (Figure 1C).

\section{Cellular uptake of the nanoparticles}

As shown in Figure 2, BEL-7402 cells were incubated with FA-MNPs and collected for FCM analysis. Higher SS intensities for BEL-7402 cells incubated with FA-MNPs were detected, suggesting NPs were inside cells, compared to that for control cells without FA-MNP treatment.

\section{Inhibition of cell proliferation}

As shown in Table 1, the inhibitive ratio of the cells cocultured with FA-MNPs did not exhibit an obvious difference compared to the sham control group. The combination of FA-MNPs and ELF-EMF significantly inhibited BEL-7402 and HL7702 cell lines (inhibitive ratios of $13.64 \% \pm 2.53 \%$ and $4.65 \% \pm 0.65 \%$, respectively, at 150 -minute exposure time point) more than ELF-EMF alone or FA-MNPs alone. Additionally, the combination of FA-MNPs and ELF-EMF inhibited the proliferation of BEL-7402 cells significantly more than HL-7702 cells.

\section{Apoptosis of BEL-7402 cells induced by EMF combined with FA-MNPs}

As shown in Figure 3, higher than $1.0 \mathrm{mg} / \mathrm{mL}$ FA-MNPs did not show further greater apoptosis effect, and so doses below $1.0 \mathrm{mg} / \mathrm{mL}$, such as $0.25 \mathrm{mg} / \mathrm{mL}$ FA-MNPs, were selected for apoptosis and proliferation-inhibition study. The results of FCM showed that more than $1.0 \mathrm{mg} / \mathrm{mL}$ FA-MNPs did not show any greater apoptosis effect (Figure 3), and thus doses below $1.0 \mathrm{mg} / \mathrm{mL}$, such as $0.25 \mathrm{mg} / \mathrm{mL}$ FA-MNPs, were selected for apoptosis and proliferation-inhibition

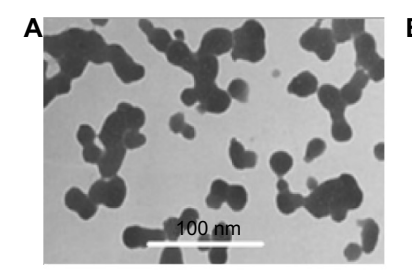

D

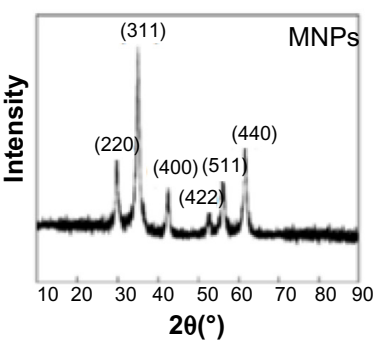

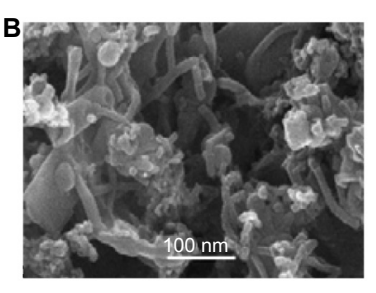

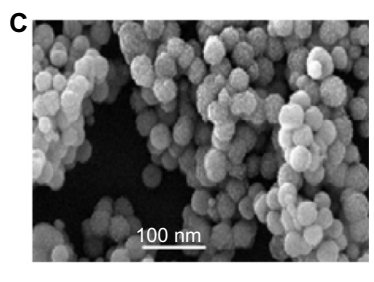

E

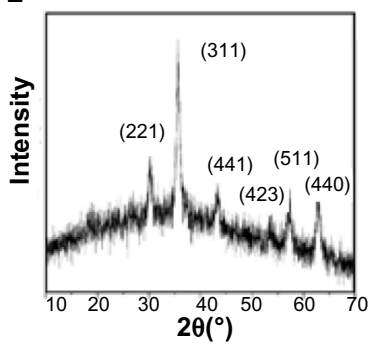

Figure I (A-E) SEM and XRD figures of MNPs and FA-MNPs. (A and D) SEM and XRD of unmodified MNPs; (B and E) SEM and XRD of lecithin-modified MNPs; (C) SEM of FA attached to lecithin-modified MNPs. Nanoscale $=100 \mathrm{~nm}$.

Abbreviations: SEM, scanning electron microscopy; XRD, X-ray diffraction; FA, folic acid; MNPs, magnetic nanoparticles. 
A

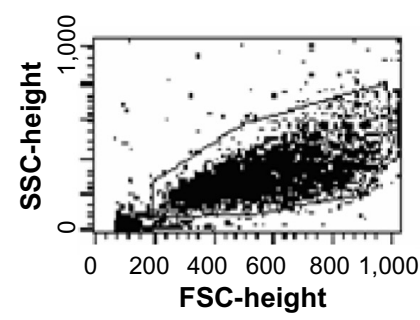

B

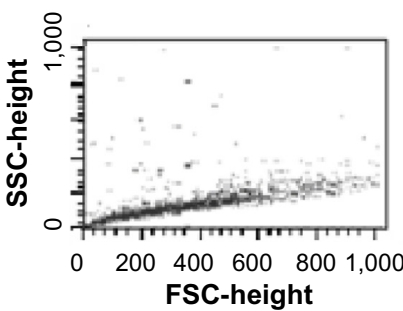

C

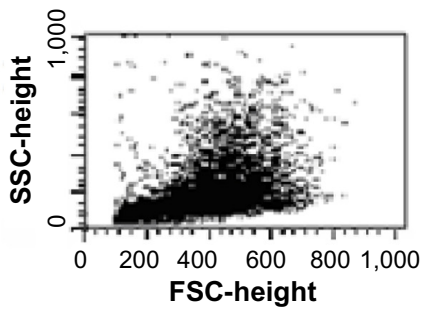

Figure 2 (A-C) Nanoparticles inside cells evaluated using flow-cytometric light-scatter analysis. (A) Gated BEL-7402 cells to avoid the cell debris and aggregated MNPs; (B) BEL-7402 cells incubated without FA-MNPs for 24 hours; (C) BEL-7402 cells incubated with FA-MNPs for 24 hours.

Abbreviations: FA, folic acid; MNPs, magnetic nanoparticles; FSC, front scatter; SSC, side scatter.

study. Table 2 further shows that FA-MNPs alone had no significant effect on the apoptosis of BEL-7402 cells or HL7702 cells compared to sham control. Addition of ELFEMF to FA-MNPs induced significantly higher apoptosis compared to FA-MNPs alone or ELF-EMF alone in both BEL-7402 cells and HL7702 cells. It should be noticed that when combined with ELF-EMF, the temperatures of the EMF-exposure and sham groups were measured, and there was no significant difference between the EMF-exposure and sham groups (data not shown). The combination also induced significantly higher apoptosis in BEL-7402 cells compared to HL7702 cells.

\section{Apoptosis is related to the types of cell lines and FA-MNP doses}

Figure 3 shows that apoptosis of BEL-7402 cells was significantly higher than that of HL7702 cells when treated with the combination of FA-MNPs with ELF-EMF. Interestingly, higher doses of FA-MNPs induced significantly higher apoptosis in both BEL-7402 cells and HL7702 cells. However, multiple comparisons showed that there was no significant difference in apoptosis ratio between $1.0 \mathrm{mg} / \mathrm{mL}$ FA-MNPs and $2.0 \mathrm{mg} / \mathrm{mL}$ FA-MNPs. Furthermore, similar results

Table I Proliferation inhibition of cells treated by folic acid-modified magnetic nanoparticles (FA-MNPs) at different concentrations for 24 hours and then combined with an extremely low-frequency electromagnetic field (ELF-EMF) for I50 minutes

\begin{tabular}{lcl}
\hline ELF-EMF inhibition ratio (\%) & BEL-7402 cells & HL7702 cells \\
\hline Sham control & $0.12 \pm 0.143^{\#}$ & $0.24 \pm 0.218^{\#}$ \\
FA-MNPs + ELF-EMF & $13.64 \pm 2.534^{*, \Lambda}$ & $4.65 \pm 0.646^{*}$ \\
ELF-EMF alone & $2.50 \pm 0.290^{*, \#}$ & $1.13 \pm 0.978^{*, \#}$ \\
FA-MNPs + Sham ELF-EMF & $0.31 \pm 0.154^{\#}$ & $0.45 \pm 0.336^{\#}$ \\
\hline
\end{tabular}

Notes: $* P<0.05$, significantly different compared to sham control; ${ }^{*} P<0.05$, significantly different compared to FA-MNPs + ELF-EMF; $\wedge P<0.05$, significantly different compared to HL7702 cell line. Sham control - cells treated with sham ELFEMF; ELF-EMF + FA-MNPs - cells treated with FA-MNPs and ELF-EMF; ELF-EMF alone - cells treated with ELF-EMF alone; FA-MNPs alone + sham ELF-EMF - cells treated with FA-MNPs and sham ELF-EMF for 150 minutes. were detected when the BEL-7402 cells were treated with different concentration of MNPs and then combined with ELF-EMF (Figure 4).

\section{Cellular iron uptake related to the types of cell lines and FA-MNP doses}

ICP-AES results showed that FA-MNP uptake of BEL7402 cells was significantly higher than that of HL7702 cells, and higher doses of FA-MNPs induced significantly higher iron uptake in both BEL-7402 cells and HL7702 cells (Figure 5). However, multiple comparisons showed that there was no significant difference between iron uptake at doses of $1.0 \mathrm{mg} / \mathrm{mL}$ and $2.0 \mathrm{mg} / \mathrm{mL}$ (Figure 6). Moreover, uptake of FA-MNPs was significantly higher in both treated BEL-7402 cells and HL7702 cells compared to MNP control $(P<0.05)$ (Figure 5).

\section{Curve estimation of relations between iron uptake and apoptosis}

As shown in Figure 6, regression analysis of curve estimation showed that apoptosis elicited by FA-MNPs

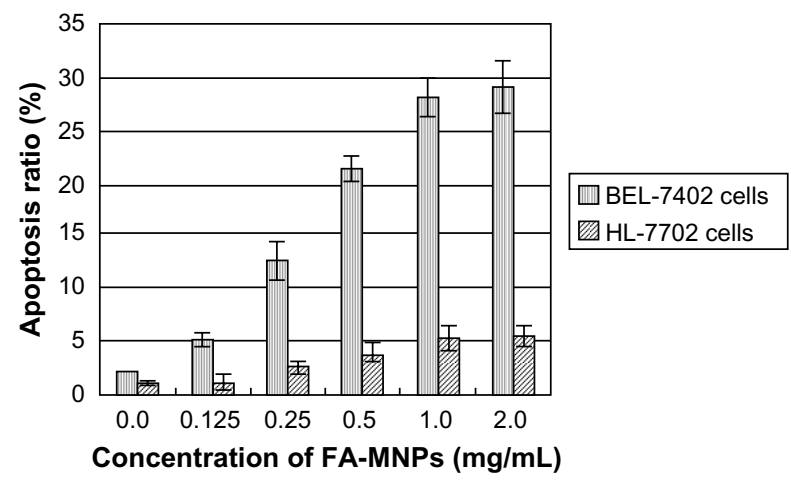

Figure 3 Apoptosis of both BEL-7402 cells and HL7702 cells treated by FA-MNPs at different concentrations for 24 hours and then combined with an ELF-EMF for 150 minutes.

Abbreviations: FA, folic acid; MNPs, magnetic nanoparticles; ELF-EMF, extremely low-frequency electromagnetic field. 
Table 2 Apoptosis of BEL-7402 and HL7702 cells treated by folic acid-modified magnetic nanoparticles (FA-MNPs) at different concentrations for 24 hours and then combined with an extremely low-frequency electromagnetic field (ELF-EMF) for 150 minutes

\begin{tabular}{lcl}
\hline ELF-EMF apoptosis ratio (\%) & BEL-7402 cells & HL7702 cells \\
\hline Sham control & $0.10 \pm 0.185^{\#}$ & $0.14 \pm 0.145^{\#}$ \\
FA-MNPs + ELF-EMF & $12.67 \pm 1.698^{*, \Lambda}$ & $2.60 \pm 0.608^{*}$ \\
ELF-EMF alone & $2.16 \pm 0.097^{\#, *}$ & $1.09 \pm 0.108^{\#, *}$ \\
FA-MNPs + Sham ELF-EMF & $0.29 \pm 0.230^{\#}$ & $0.37 \pm 0.334^{\#}$ \\
\hline
\end{tabular}

Notes: $* P<0.05$, significantly different compared to ELF-EMF alone; ${ }^{*}<0.05$, significantly different compared to FA-MNPs + ELF-EMF; $\wedge P<0.05$, significantly different compared to HL7702 cell line. Sham control - cells treated with sham ELFEMF; ELF-EMF + FA-MNPs - cells treated with FA-MNPs and ELF-EMF; ELF-EMF alone - cells treated with ELF-EMF alone; FA-MNPs alone + sham ELF-EMF - cells treated with FA-MNPs and sham ELF-EMF for 150 minutes.

was proportional to the cellular iron uptake of FA-MNPs $\left(F=96.65, P=1.16 \times 10^{-8}\right)$.

\section{Discussion}

Our previous study showed that unmodified MNP coculturing did not affect cell proliferation compared to control cells. However, unmodified MNPs combined with ELF-EMF significantly inhibited cell proliferation and induced early apoptosis compared to MNPs or ELF-EMF alone. ${ }^{9}$ FA coatings of MNPs could be used to facilitate uptake to cancer cells expressing the folate receptor for cancer therapy. ${ }^{16,17}$ In this experiment, we prepared FA-coated MNPs and found that these also showed similar effects of cell-proliferation inhibition and cell-apoptosis induction. FA-MNPs (at doses of $0-2 \mathrm{mg} / \mathrm{mL}$ ) alone showed no effect on cell-proliferation inhibition or apoptosis induction of BEL-7402 cells and HL7702 cells. However, when combined with ELF-EMF, FAMNPs significantly inhibited cell proliferation and induced cell apoptosis. Higher doses of FA-MNPs obtained a greater

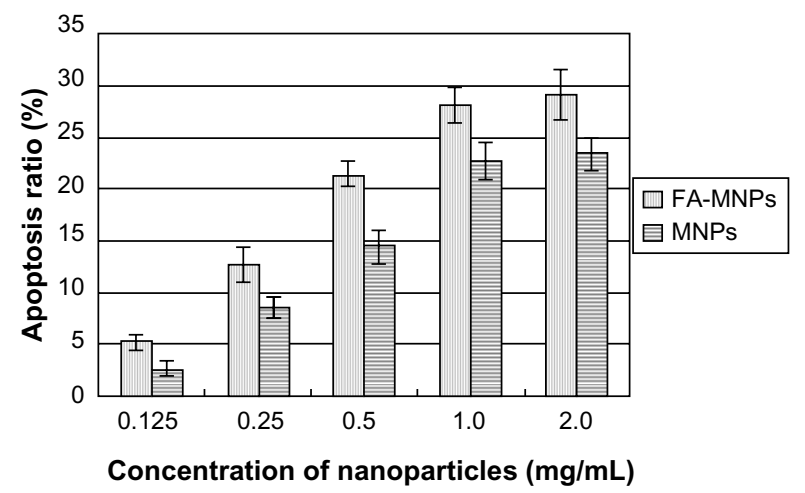

Figure 4 Apoptosis of BEL-7402 cells treated by FA-MNPs versus MNPs at different concentrations for 24 hours and then combined with an ELF-EMF for 150 minutes.

Abbreviations: FA, folic acid; MNPs, magnetic nanoparticles; ELF-EMF, extremely low-frequency electromagnetic field. apoptosis effect, but when the concentration of FA-MNPs was more than $1.0 \mathrm{mg} / \mathrm{mL}$, there was no significant difference between them. Furthermore, the apoptosis-induction effect was significantly better in liver cancer BEL-7402 cells compared to normal liver HL 7702 cells. We noted that these kind of relations between apoptosis and FA-MNP doses (Figure 3) were similar to the relations between FA-MNP uptake and FAMNP doses (Figure 4). Iron uptake by BEL-7402 cells was also significantly higher than that by HL7702 cells. Though the higher dose of FA-MNPs resulted in the higher intracellular iron uptake, the intracellular uptake of iron reached a peak when the dose of FA-MNPs was more than $1.0 \mathrm{mg} / \mathrm{mL}$. This suggested that apoptosis may not be proportional to the FA-MNP doses but to FA-MNP cellular uptake, which was further demonstrated by the regression analysis that apoptosis is linear to cellular FA-MNP uptake.

It was reported that curcumin-loaded magnetic NPs showed a preferential uptake in MDA-MB-231 cells in a concentration-dependent manner. ${ }^{18}$ Also, our study showed that FA-MNPs showed a dose-dependent preferential iron uptake in BEL-7402 cells; correspondingly, FA-MNPs elicited selective apoptosis in BEL-7402 cells in a seemingly concentration-dependent manner. However, $2.0 \mathrm{mg} / \mathrm{mL}$ NPs could not bring higher apoptosis or iron uptake compared to $1.0 \mathrm{mg} / \mathrm{mL}$ FA-MNPs, suggesting that there may exist a saturated status for cellular particle uptake in either BEL-7402 cells or HL7702 cells. Doses higher than $1.0 \mathrm{mg} / \mathrm{mL}$ did not bring further higher iron uptake per cell. This suggests that the actual apoptosis elicited by FA-MNPs and ELF-EMF may not be concentration-dependent, but uptake-dependent. To further demonstrate that the FA coatings play an important role in facilitating cellular FA-MNPs uptake, we compared the FA-MNPs to unmodified MNPs in cellular uptake. Our experiment showed that FA-MNPs produced better iron uptake compared to MNPs in BEL-7402 cells. Therefore, higher expression of FA receptors on BEL-7402 cells may promote the uptake of FA-MNPs rather than MNPs. ${ }^{19}$ High expression of folate receptors on BEL-7402 cells may explain the higher uptake of FA-MNPs in BEL-7402 cells compared to HL7702 cells; however, BEL-7402 cells also showed higher uptake of iron compared to HL7702 cells (Figure 5), suggesting that higher uptake of particles may not only depend on folate receptor-mediated endocytosis but also on other pathways. Noticeably, FA-MNPs showed significantly higher iron uptake compared to MNP-treated BEL-7402 cells, but not in HL7702 cells. Therefore, though only four replicates were not enough to reveal the significance of iron uptake between FA-MNPs and MNP-treated HL7702 


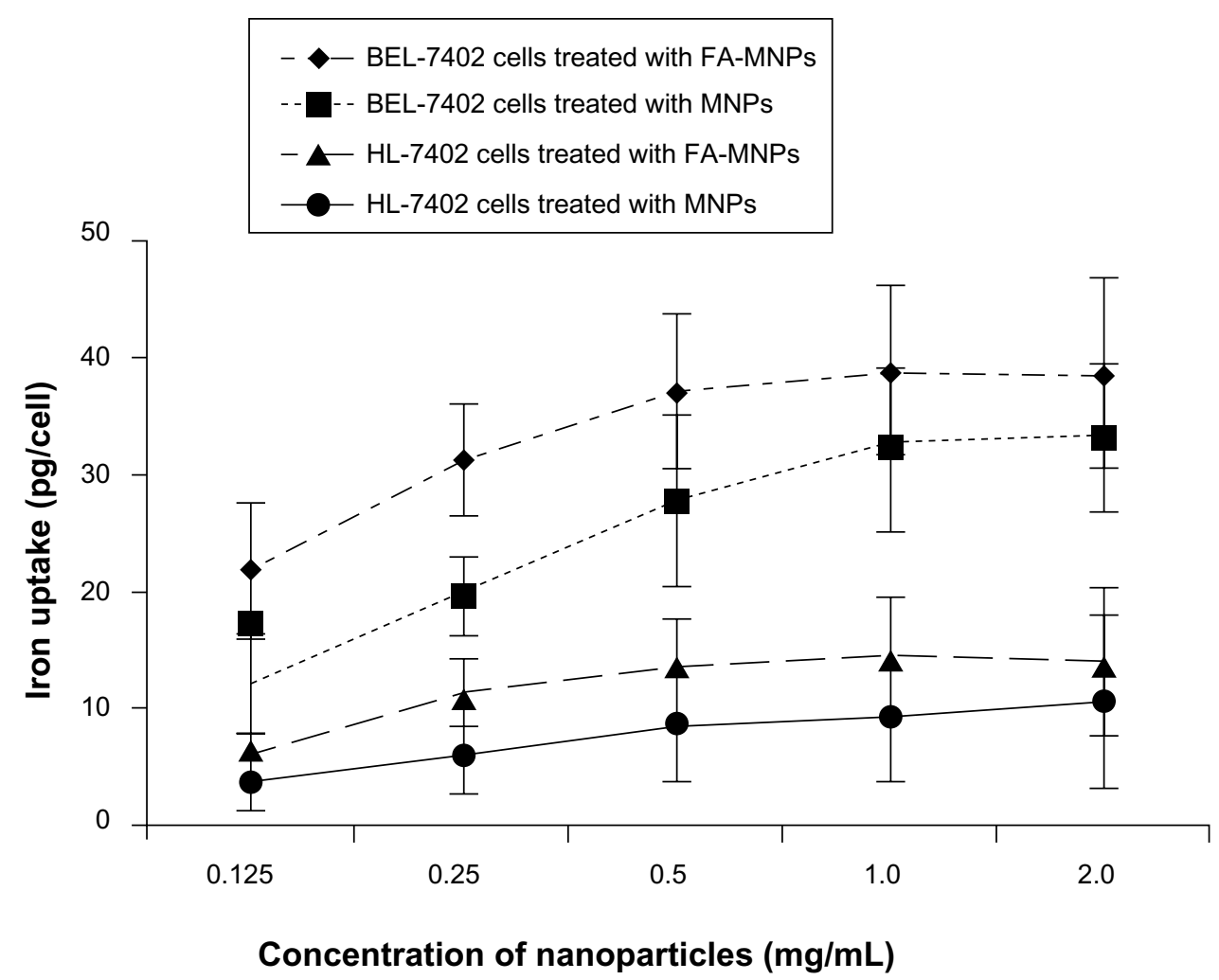

Figure 5 Uptake of MNPs in both BEL-7402 cells and HL770 cells treated by either FA-MNPs or MNPs at different concentrations for 24 hours and then combined with an ELF-EMF for 150 minutes.

Abbreviations: FA, folic acid; MNPs, magnetic nanoparticles; ELF-EMF, extremely low-frequency electromagnetic field.

cells, it can be demonstrated that BEL-7402 cells with high expression of folate receptors took up more FA-MNPs than MNPs, while HL7702 cells with low expression of folate receptors did not.

As a result, it could be inferred that the selective FA-MNP uptake by BEL-7402 cells due to the higher expression of FA receptors on the BEL-7402 cells may be the reason for the selective induction of apoptosis in BEL7402 liver cancer cells. ${ }^{19}$ However, the coating process may change the size,

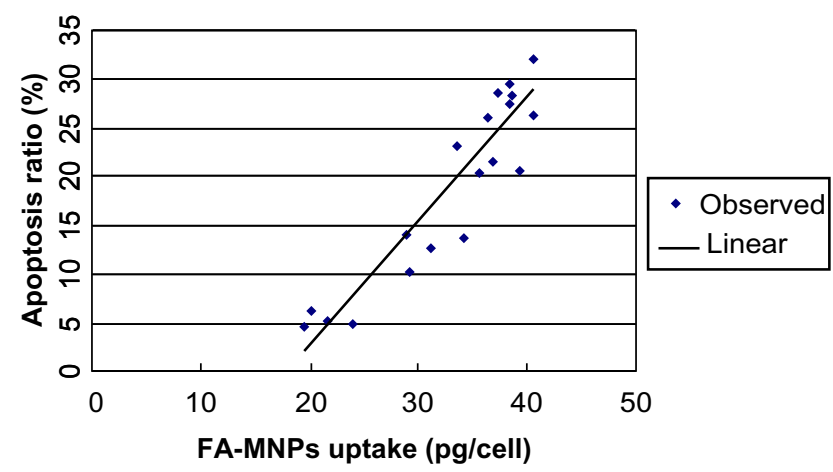

Figure 6 Regression analysis of the relationship between the uptake of FA-MNPs and apoptosis.

Notes: $F=96.65 ; P=1.16 \times 10^{-8}$.

Abbreviations: FA, folic acid; MNPs, magnetic nanoparticles. morphology, or other characteristics of MNPs. As the SEM images show, the modification of FA changed the shape of the MNP particles remarkably, and the FA-MNPs became irregular. This may be also related to the higher cellular uptake and greater apoptosis-induction effect preferentially in BEL-7402 cells. Moreover, the characterization of MNPs has been done in deionized water. Interaction with the serum proteins within the culture medium may dramatically affect MNP dispersion, and thus may affect the efficiency of MNP uptake into cells. ${ }^{20}$ Several studies have demonstrated that certain structural components of FA are necessary for binding and internalization; conjugating FA to MNPs may hide the structural components necessary for internalization, and thus affect FA receptormediated endocytosis. ${ }^{21,22}$ Free FA competition studies using FR-positive/negative cell lines need to be performed in the future to confirm FR-mediated receptor uptake.

\section{Conclusion}

These results indicate that FA-MNPs combined with ELFEMF treatment showed a selective apoptosis-induction effect in liver cancer BEL-7402 cells in an FA-MNP uptakedependent manner. This may provide a new therapeutic approach for HCC patients in the future. 


\section{Acknowledgments}

This work was supported by the Nature and Science Fund (Project 81000997), National High Technology Research and Development Program of China (Program 863, 2007AA0222006), National Nature Science Foundation of China (30740062, 30872970), National High Technology Research and Development Program 863 Projects of the People's Republic of China (2012AA022703), and the National Nature Science Foundation of the People's Republic of China (81370673).

\section{Disclosure}

The authors report no conflicts of interest in this work.

\section{References}

1. Jemal A, Ward E, Hao Y, Thun M. Trends in the leading causes of death in the United States, 1970-2002. JAMA. 2005;294:1255-1259.

2. Bosch FX, Ribes J, Díaz M, Cléries R. Primary liver cancer: worldwide incidence and trends. Gastroenterology. 2004;127:S5-S16.

3. Forner A, Llovet JM, Bruix J. Hepatocellular carcinoma. Lancet. 2012;379:1245-1255.

4. Ansary A, Faddah LM. Nanoparticles as biochemical sensors. Nanotechnol Sci Appl. 2010;3:65-76.

5. Wang J, Chen Y, Chen B, et al. Pharmacokinetic parameters and tissue distribution of magnetic $\mathrm{Fe}_{3} \mathrm{O}_{4}$ nanoparticles in mice. Int J Nanomedicine. 2010;5:861-866.

6. Sonvico F, Mornet S, Vasseur S, et al. Folate-conjugated iron oxide nanoparticles for solid tumor targeting as potential specific magnetic hyperthermia mediators: synthesis, physicochemical characterization, and in vitro experiments. Bioconjug Chem. 2005;16:1181-1188.

7. Wu W, Chen B, Cheng J, et al. Biocompatibility of $\mathrm{Fe}_{3} \mathrm{O}_{4} / \mathrm{DNR}$ magnetic nanoparticles in the treatment of hematologic malignancies. Int $J$ Nanomedicine. 2010;5:1079-1084.

8. Repacholi MH, Greenebaum B. Interaction of static and extremely low frequency electric and magnetic fields with living systems: health effects and research needs. Bioelectromagnetics. 1999;20:133-160.
9. Chen ZQ, Wen J, Tu WY, Xiao L, Fang Z. A study on early apoptosis of hepatoma Bel-7402 cells in vitro treated by altering-electric magnetic field exposure of extremely low frequency combined with magnetic nano- $\mathrm{Fe}_{3} \mathrm{O}_{4}$ powders. Appl Mech Mater. 2013;364:742-748.

10. Hu S, Zhang Y. Endostar-loaded PEG-PLGA nanoparticles: in vitro and in vivo evaluation. Int J Nanomedicine. 2010;5:1039-1048.

11. Bhattacharya R, Patra CR, Earl A, et al. Attaching folic acid on gold nanoparticles using noncovalent interaction via different polyethylene glycol backbones and targeting of cancer cells. Nanomedicine. 2007;3: 224-238.

12. Lowry MB, Duchemin AM, Robinson JM, Anderson CL. Functional separation of pseudopod extension and particle internalization during Fc gamma receptor-mediated phagocytosis. J Exp Med. 1998;187: 161-176.

13. Quintana A, Raczka E, Piehler L, et al. Design and function of a dendrimer-based therapeutic nanodevice targeted to tumor cells through the folate receptor. Pharm Res. 2002;19:1310-1316.

14. Moore A, Basilion JP, Chiocca EA, Weissleder R. Measuring transferrin receptor gene expression by NMR imaging. Biochim Biophys Acta. 1998;1402:239-249.

15. Suzuki H, Toyooka T, Ibuki Y. Simple and easy method to evaluate uptake potential of nanoparticles in mammalian cells using a flow cytometric light scatter analysis. Environ Sci Technol. 2007;41: 3018-3024.

16. Saltan N, Kutlu HM, Hur D, Iscan A, Say R. Interaction of cancer cells with magnetic nanoparticles modified by methacrylamido-folic acid. Int J Nanomedicine. 2011;6:477-484.

17. Antony AC. The biology chemistry of folate receptors. Blood. 1992;79:2807-2820.

18. Yallapu MM, Othman SF, Curtis ET, et al. Curcumin-loaded magnetic nanoparticles for breast cancer therapeutics and imaging applications. Int J Nanomedicine. 2012;7:1761-1779.

19. Zhang J, Deng D, Qian Z, et al. The targeting behavior of folate-nanohydrogel evaluated by near infrared imaging system in tumor-bearing mouse models. Pharm Res. 2010;27:46-55.

20. Wiogo HT, Lim M, Bulmus V, Gutiérrez L, Woodward RC, Amal R. Insight into serum protein interactions with functionalized magnetic nanoparticles in biological media. Langmuir. 2012;28:4346-4356.

21. Pelkmans L, Helenius A. Endocytosis via caveolae. Traffic. 2002;3:311-320.

22. Huennekens FM, Vitols KS, Pope LE, Fan J. Membrane transport of folate compounds. J Nutr Sci Vitaminol (Tokyo). 1992;Spec No: 52-57.
International Journal of Nanomedicine

\section{Publish your work in this journal}

The International Journal of Nanomedicine is an international, peerreviewed journal focusing on the application of nanotechnology in diagnostics, therapeutics, and drug delivery systems throughout the biomedical field. This journal is indexed on PubMed Central, MedLine, CAS, SciSearch $\AA$, Current Contents $₫ /$ Clinical Medicine,

\section{Dovepress}

Journal Citation Reports/Science Edition, EMBase, Scopus and the Elsevier Bibliographic databases. The manuscript management system is completely online and includes a very quick and fair peer-review system, which is all easy to use. Visit http://www.dovepress.com/ testimonials.php to read real quotes from published authors. 